\title{
Estudo da variabilidade da cobertura de nuvens altas na Amazônia Central
}

\author{
Maria Aparecida Senaubar ALVES ${ }^{1}$, Luiz Augusto Toledo MACHADOㄹ, \\ Gannabathula Sri Sesha Durga PRASAD 2
}

\begin{abstract}
RESUMO
Foram estudados os fenômenos meteorológicos que ocorrem na Amazônia Central (Manaus) utilizando-se um conjunto de imagens de satélites, com o objetivo de identificar as oscilaçōes que mais contribuem para a variabilidade da cobertura de nuvens, e verificar se há modificações nestas oscilações em anos de El Niño e La Niña. O ciclo anual e o ciclo semi-anual são os principais responsáveis pela variabilidade da cobertura de nuvens altas na região. As oscilações interanuais associadas aos fenômenos El Niño/La Niña também contribuem fortemente para a variabilidade total da cobertura de nuvens altas. As oscilações intra-sazonais e interdiurnas apresentam uma variabilidade menor. Estendendo-se a análise para uma região compreendida de $1,5^{\circ} \mathrm{S}$ a $6^{\circ} \mathrm{S}$ e $68^{\circ} \mathrm{W}$ a $54^{\circ} \mathrm{W}$, centrados em Manaus, observa-se que os períodos de 60, 45 e 30 dias possuem maior porcentagem de potência a leste de Manaus; o período de 20 dias possui maior porcentagem de potência no centro (próximo de Manaus); e os períodos de 8, 6 e 4 dias possuem maior porcentagem de potência a oeste de Manaus. As frentes frias que alcançam a janela de $10^{\circ}$ de longitude e $2,5^{\circ}$ de latitude centrada em Manaus, modulam de alguma forma a atividade convectiva na região de Manaus atuando como uma forçante das oscilaçōes interdiurnas (47\% dos casos), das oscilaçōes intra-sazonais, (15\%) e das oscilações de 55 a 65 dias (13\%). Convém ressaltar que não necessariamente as frentes frias causaram as oscilações. As frentes podem favorecer ou induzir a convecção no local.
\end{abstract}

PALAVRAS-CHAVE

Oscilações, Cobertura de nuvens, Convecção, Amazônia Central.

\section{Study of the variability of high cloud covering over central Amazon region}

\section{ABSTRACT}

The meteorological phenomena that occur in the Central Amazonia (Manaus) are studied from ISCCP-C1 Satellite-based data. The oscillations that contribute more significantly to the cloud cover variability and its relation to El Niño and La Niña events case identified. The annual and semi-annual oscillation times scales are the main responsible for the variability of the high cloud cover over Manaus. The inter-annual oscillations associated with El Niño and La Niña events also contribute significantly to the total variability of the high cloud cover, while intraseasonal and day-to-day oscillations show a reduced contribution. However, the intraseasonal and day-to-day oscillations are important since they do influence the predictability on short and medium range forecast. The studied region was extended to 21 sections, centered in Manaus. The 60, 45 and 30 day-oscillations exhibited a higher spectral power density percentage over eastern Manaus; the 20 day-oscillations, over the central Manaus; and the 8, 6 and 4 day-oscillations, over western Manaus. The cold fronts that reach central Amazonia modulate convective activity over Manaus region, acting as a forcing for day-to-day oscillations (47\% of the cases), intraseasonal oscillations (15\%), and 55 to 65 day-oscillations (13\%). It is important to point out that the cold fronts did not necessarily establish the oscillations, but they may have favored or induced convection over Manaus region.

\section{KEY-WORDS}

Oscillation, clouds cover, convection, central Amazon

\footnotetext{
${ }^{1}$ Instituto de Aeronáutica e Espaço, Divisão de Ciências Atmosféricas, Pça Marechal Eduardo Gomes, 50, 12228-904, São José dos Campos, SP. Tel: (12) 3947-4590, Email: cida@iae.cta.br. ${ }^{2}$ Instituto Nacional de Pesquisas Espaciais, Av. dos Astronautas n ${ }^{0}$ 1758, São José dos Campos, SP.
} 


\section{INTRODUÇÃO}

A Amazônia caracteriza-se por possuir um alto conteúdo de vapor d'água na atmosfera, fortes fluxos de calor latente provenientes da superfície e elevado índice pluviométrico. A precipitação e a cobertura de nuvens são os elementos climáticos com maior variabilidade espacial e temporal na região tropical (Fisch et al., 1998). Sabendo-se que as nuvens exercem um papel significativo sobre as trocas de energia entre as várias escalas de tempo dos fenômenos atmosféricos, surge o interesse de conhecer com maiores detalhes a periodicidade dos sistemas atmosféricos que atuam na região Amazônica e as interaçōes entre as escalas dos mesmos.

Os mecanismos dinâmicos que produzem precipitação na Amazônia, descritos por Vianello e Alves (1991), resultam da combinação ou da atuação predominante da Zona de Convergência Intertropical (ZCIT), das linhas de instabilidade, da penetração de sistemas frontais, da fonte de vapor d'água representada pela floresta amazônica e pela atuação da cordilheira dos Andes.

Oliveira (1986), utilizando imagens de satélites meteorológicos, verificou que os sistemas frontais freqüentemente organizam e interagem com a convecção sobre o Brasil tropical. Molion (1987) sugere que a variabilidade da precipitação pode ser parcialmente devida à zona de convergência persistente originadas de ou associadas com sistemas frontais, que organizam convecção profunda sobre o Brasil Tropical.

Chapa et al. (1998), utilizando índices convectivos derivados de imagens do METEOSAT, estudaram as principais periodicidades associadas à atividade convectiva na América do Sul para o período de novembro de 1994 a maio de 1995. Os autores identificaram próximo do Equador periodicidades em torno de aproximadamente 5 a 8 dias durante todo o ano, e em torno de 20 dias entre $4,5^{\circ} \mathrm{N}$ e $22,5^{\circ} \mathrm{S}$ principalmente durante o outono e inverno.

Segundo Matthews (2000) a oscilação 30-60 dias, conhecida como oscilação de Madden-Julian, consiste de uma onda atmosférica planetária (número de onda zonal 1-2) cuja propagação para leste, ao longo da faixa equatorial global, ocasiona variaçōes significativas nos padrōes da circulação atmosférica e convecção tropical. Segundo Kayano e Kousky (1992) a oscilação 30-60 dias se propaga de forma relativamente uniforme nos oceanos Índico e Pacífico equatorial oeste. No restante do domínio tropical, a sua propagação não é uniforme e as anomalias são menos pronunciadas, porém detectáveis.

$\mathrm{Na}$ América do Sul, a oscilação de 30-60 dias tem sido associada às variações na posição e na intensidade da Zona de Convergência do Atlântico Sul (ZCAS), com flutuações de escala global na circulação da alta troposfera e na convecção tropical (Casarin e Kousky, 1986). Kayano \& Kousky (1999), utilizando dados de reanálise NCEP/NOAA no período de 1979 a 1995 , estudaram a evolução da oscilação de 30-60 dias durante o verão e inverno austral. Em ambas as estações, o padrão encontrado foi o deslocamento da convecção tropical para leste com período de 45 dias. No verão, a oscilação de 30-60 dias apresenta fortes variabilidades regionais, indicando a interação dessa oscilação com a atividade convectiva de certas áreas como a da Zona de Convergência do Pacífico Sul (ZCPS) e a do nordeste da América do Sul.

Nascimento \& Brito (2002) utilizando dados de pressão média diária em Fortaleza, no período de La Niña (os meses de agosto a novembro de 1985 e de setembro a dezembro de 1986) e de El Niño (os meses de outubro de 1992 até dezembro de 1993) observaram a atuação da oscilação de 30-60 dias em ambos os conjuntos de dados.

A precipitação é fortemente influenciada pela variabilidade interanual da circulação geral da atmosfera. Eventos de El Niño no Pacífico parecem diminuir os totais pluviométricos na Amazônia, enquanto durante anos de La Niña se observa anomalia positiva de precipitação (Cutrim et al., 2000).

Marengo et al. (2001), analisando os dados de precipitação, reanálises do NCEP, ECMWF e OLR do início e final da estação chuvosa da Bacia Amazônica $\left(5^{\circ} \mathrm{N}-15^{\circ} \mathrm{S}, 50^{\circ}-72.5^{\circ} \mathrm{W}\right)$ no período de 1979-1996 mostraram alta correlação entre as anomalias de temperatura da superfície do mar (TSM) do Atlântico e Pacífico Tropical com a duração da estação chuvosa. Quando ocorre anomalia positiva de TSM no Pacífico, favorece uma estação chuvosa curta na Amazônia. Entretanto, quando ocorre anomalia positiva no Atlântico, favorece uma estação chuvosa longa na mesma região.

Portanto, os resultados dos trabalhos anteriores forneceram evidências de que a região Amazônica é fortemente influenciada por oscilaçóes interdiurnas, intra-sazonais e interanuais e, conseqüentemente, por interaçôes entre estas diferentes escalas. Desta forma, o objetivo deste trabalho é analisar a importância relativa destas escalas temporais, e estudar como essas oscilações modulam a convecção da região central da Amazônia em anos de El Niño e La Niña.

\section{MATERIAL E MÉTODOS}

Neste trabalho foram utilizados dados do Internacional Satellite Cloud Climatology Project (ISCCP) no formato ISCCPC1, para o período de julho de 1983 a agosto de 1994. Os dados ISCCP-C1 são derivados de imagens dos satélites meteorológicos geoestacionários e de órbita polar, sendo disponibilizados a cada 3 horas $(00,03,06,09,12,15,18$ e 21 UTC), e fornecendo uma estatística sobre um setor de $2,5^{\circ} \mathrm{x}$ $2,5^{\circ}$ de latitude e longitude. As informações básicas disponíveis são os números totais de pixels no setor e o número de pixels associado a diferentes alturas de topos de nuvens. Os detalhes utilizados para a obtenção destes dados estão descritos em Schiffer 
\& Rossow (1983), Rossow et al. (1991) e Rossow e Walker (1991).

Inicialmente, analisa-se um setor da imagem ISCCP-C1 centrado em $3,5^{\circ} \mathrm{S}$ e $61,25^{\circ} \mathrm{W}$. Para o setor, obtém-se uma série temporal de informações sobre a cobertura de nuvens formada por cerca de 32000 dados ( 8 imagens por dia $\times 365$ dias por ano $\mathrm{x} 11$ anos). Este setor compreende a região de Manaus.

São utilizados três tipos de cobertura de nuvens. O primeiro tipo é a cobertura total de nuvens do setor, representada como a razão entre o número de pixels coberto por nuvens e o total de pixels do setor. O segundo tipo é a cobertura de nuvens altas do setor, definida como a razão entre o número de pixels coberto por nuvens com topo superior a $560 \mathrm{hPa}$ (aproximadamente $4.800 \mathrm{~m}$ ) e o número total de pixels do setor. O terceiro tipo éa cobertura de nuvens associada à atividade convectiva, calculada como a razão entre o número de pixels coberto por nuvens com topo acima de $310 \mathrm{hPa}$ (aproximadamente $9.100 \mathrm{~m}$ ) e o número total de pixels do setor.

Guedes e Machado (1997) verificaram que o horário de 00:00 UTC possui máxima cobertura de nuvens altas na América do Sul. Assim, com o objetivo de eliminar a oscilação diurna e destacar as outras oscilações utiliza-se apenas o horário de 00:00 UTC para a análise das oscilações interanual, anual, semi-anual, intra-sazonal e interdiurnas.

A ferramenta matemática utilizada foi a Transformada Wavelet de Morlet (TWM). A TWM permite identificar objetivamente as oscilaçōes no domínio tempo-freqüência, além de auxiliar na análise na interação entre as diferentes escalas temporais.

O termo "Wavelet" refere-se a um conjunto de pequenas ondas formadas por dilataçōes e translaçōes de uma função básica, $\Psi(\mathrm{t})$, chamada de Wavelet mãe, que possui energia finita e média igual a zero (Weng \& Lau, 1994). Em cada função básica, a localização temporal é determinada pelo parâmetro de translação e a localização em freqüência pelo parâmetro de dilatação. A Wavelet de Morlet é uma Wavelet complexa; sendo que os coeficientes da parte real e imaginária fornecem a amplitude, a fase e a potência (quadrado do módulo da amplitude). A Wavelet de Morlet utilizada é um produto de uma gaussiana por uma senóide e fornece informaçôes de alta resolução que permite avaliar a variabilidade do sinal em tempo e freqüência. A Wavelet de Morlet pode ser expressa por:

$$
\Psi(t)=e^{i w t} e^{-(2 / 2)}
$$

Onde:

$i=\sqrt{-1}, w=6.0$ é a freqüência adimensional segundo Farge (1992), e té o tempo.
Em virtude dos coeficientes apresentarem uma forma canônica, as TWM têm uma limitação no início e no final do domínio temporal de estudo. Deste modo, alguma informação é perdida nos extremos das estruturas em escalas maiores.

A série temporal apresenta algumas faltas de imagens de satélites. Essas falhas na série foram da ordem de $1 \%$. Portanto, utiliza-se para os casos de período de falhas menores que um dia, uma interpolação linear com o vizinho mais próximo usando a ferramenta Matlab (1995).

\section{RESULTADOS E DISCUSSÃO}

Para compreender melhor a climatologia da cobertura de nuvens da região em estudo, são feitas algumas análises preliminares utilizando os três tipos de cobertura de nuvens: cobertura total, nuvens altas e nuvens convectivas.

A Figura 1 apresenta a média mensal dos diferentes tipos de cobertura de nuvens no setor centrado em Manaus para os anos de 1983 a 1994. A porcentagem da cobertura total de nuvens varia de $81 \%$ a $48 \%$; de nuvens altas, de $72 \%$ a $28 \%$; e de nuvens associadas à atividade convectiva, de $9 \%$ a $36 \%$. Observase uma forte variação sazonal para todos os tipos de cobertura de nuvens, com o máximo ocorrendo entre fevereiro e março, e o mínimo, entre julho e agosto. Em média, o período com maior quantidade de nuvens altas se encontra entre os meses de outubro a maio, com mínimo entre julho a setembro. Este resultado concorda com Rao \& Hada (1990) e Fisch et al. (1998). Guedes \& Machado (1997) observaram que há uma desintensificação da convecção sobre os oceanos e deslocamento da ZCIT para o sul, do inverno para verão, seguida de um forte aumento da convecção sobre o continente. No outono, observou-se um padrão semelhante ao da primavera, exceto na regiáo do Atlântico Tropical e na costa norte-nordeste do Brasil, onde existe um significativo aumento da nebulosidade.

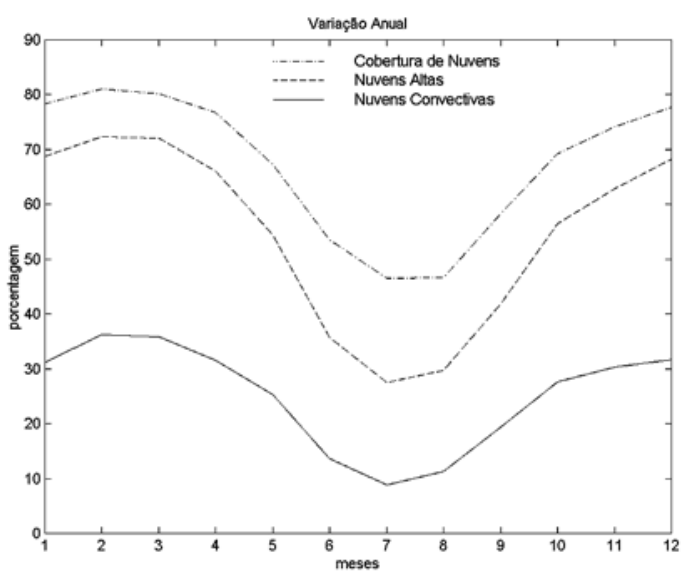

Figura 1 - Média mensal da cobertura total de nuvens, de nuvens alta e de nuvens convectiva para um setor de $2,5^{\circ} \times 2,5^{\circ}$, centrado em Manaus, para os anos de 1983 a 1994. Na ordenada tem-se os meses e, na abscissa, a porcentagem da cobertura de nuvens. 
A Figura 2 apresenta o desvio da média mensal dos três tipos de cobertura de nuvens em função do tempo. Existe uma forte variabilidade interanual. Os anos com eventos de El Niño coincidem, em geral, com os anos com desvio negativo. A cobertura de nuvens abaixo da média sugere índices pluviométricos abaixo da média. Uma exceção à regra é o El Niño de 86/87, que apresenta desvio positivo. O ano de 1987 é um ano de transição, de desvios positivos da cobertura de nuvens para desvios negativos.

Coelho et al. (1999) realizaram um estudo climatológico sazonal de precipitação sobre o Brasil durante eventos classificados como fortes, moderados e fracos de El Niño e La Niña. Os autores obtiveram alguns resultados que indicam, num dado trimestre, distinçōes nas características do padrão de precipitação entre os eventos forte a moderado e fraco. Os El Niños de 82/83 e 91/92 e as La Niñas 85/86 e 88/89 foram classificados como fortes a moderados; os El Niños de 86/87 e 93/94 e as La Niñas de 84/ 85 , como fracos.

Embora o ano de 86/87 tenha sido considerado como El Niño fraco por Coelho et al. (1999), outros autores (Cavalcanti, 1996; Kousky, 1987), documentaram que a condição de El Niño só começou atuar em janeiro de 1987. Isso explica porque, a partir do início de 1987, o valor do desvio começa a diminuir.

De uma forma geral, os anos de La Niña correspondem a desvios positivos, isto é, cobertura de nuvens acima da média, o que sugere índices pluviométricos também acima da média. Embora o ano de 1988 tenha sido considerado como um evento forte de La Niña por Coelho et al. (1999), foram observados (Figura 2) desvios negativos da cobertura de nuvens. Segundo Oliveira (1999), o episódio de La Niña 88/89 teve início em abril de 1988 e perdurou até abril de 1989, e o trimestre de

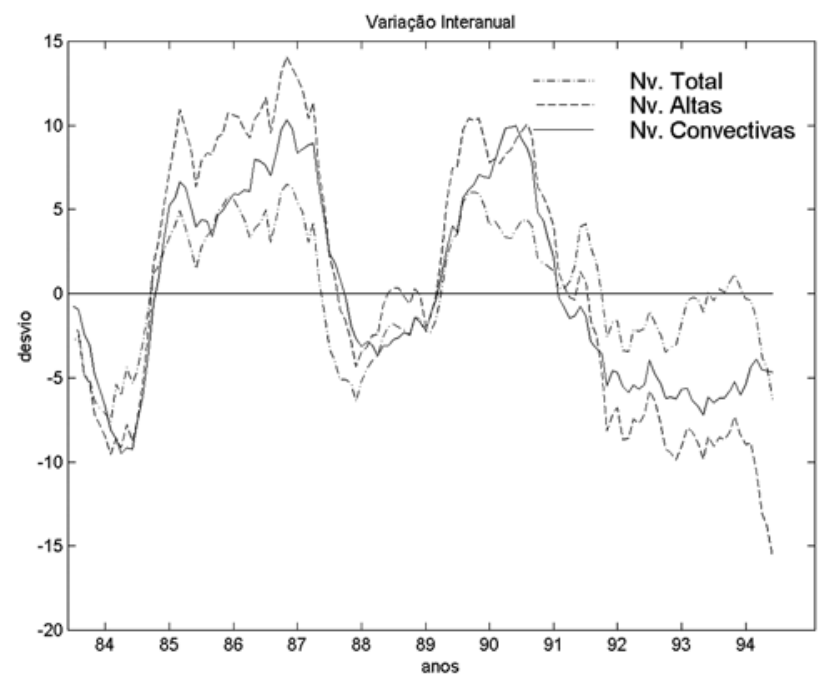

Figura 2 - Série temporal do desvio da média mensal da cobertura total, altas e convectivas de nuvens suavizada com um filtro de 6 meses, para um setor de $2,5^{\circ} \times 2,5^{\circ}$, centrado em Manaus. maiores anomalias de chuva deu-se de março a maio de 1989. Isso explica porque, no início de 1989, os valores dos desvios tornam-se menos negativos. Logo, apesar da existência de um padrão geral de variabilidade interanual da cobertura de nuvens associado aos eventos El Niño (abaixo da média) e La Niña (acima da média), existem outros fatores climáticos que podem modificar este padrão.

$\mathrm{Na}$ Figura 2, nota-se também que os maiores desvios foram obtidas para a cobertura de nuvens altas, isso evidencia que os processos associados à convecção são os que mais sofrem influências interanuais. Desta forma, as nuvens altas são escolhidas em função da maior variabilidade interanual.

Segundo Andreoli et al. (2004), as secas severas no NEB, objeto de inúmeros estudos por seus impactos sociais e econômicos, tem sido relacionada à ocorrência do fenômeno do El Niño. Esta hipótese é baseada no fato de que para alguns anos de El Niño (fortes ou moderados), notavelmente, 1877-1878, 1891, 1900, 1907, 1932, 1941, 1958, 1983, Fortaleza, no NEB, sofreu secas severas. Sob condições de El Niño, a circulação de Walker é deslocada para leste, tal que seu ramo ascendente localiza-se sobre o oceano Pacífico central e leste onde a convecção é intensificada, e seu ramo descendente localiza-se sobre o NEB e Atlântico Tropical adjacente, onde a convecção é inibida. Entretanto, a relação entre El Niño e as secas no NEB não é unívoca, alguns autores mostraram que dos $46 \mathrm{El} \mathrm{Niño} \mathrm{(fortes} \mathrm{e}$ moderados) do período de 1849-1992, somente 21 (45\%) estiveram associados à secas severas em Fortaleza. Convém lembrar que as células de Walker são células de circulação zonal, definidas na região equatorial, que se organizam entre os continentes e os oceanos. Estão associadas ao aquecimento diferencial das massas continentais, em relação às massas oceânicas. Nas células de Walker ocorre ascensão sobre os continentes (mais quentes) e subsidência sobre os oceanos (mais frios), de modo particular nas regiôes orientais do Pacífico e do Attântico. Normalmente são utilizadas para explicar eventos climáticos extremos, como El Niño e seu oposto La Niña. (Oliveira et al., 2001).

A Figura 3 apresenta a parte real dos coeficientes da Wavelet, onde mostra os principais períodos em função do tempo. Foram observadas algumas características que podem ser associadas à presença de El-Niño ou La Niña que ocorreram durante os anos analisados. Observando-se entre os períodos de 1.097-2.195 dias encontra-se uma oscilação que coincide com a presença de El-Niño nos anos de 1983, 1987-1988 e 1992-1994 e La Niña nos anos de 1984-1986, 1989-1991. Observa-se também que existe interação de escalas em alguns anos de El Niño e La Niña. Este fato evidência a importância desta flutuação interanual na variabilidade da cobertura de nuvens altas na Amazônia. Verifica-se que o período predominante é a oscilação anual que está entre os períodos de 548-274 dias. Uma outra ocorrência que também pode ser identificada é a oscilação semi-anual que aparece próximo do período de 274-137 dias. Esta oscilação é provavelmente resultado da nebulosidade advinda da migração 
SE/NW da convecção amazônica e também devido à localização da Alta da Bolívia (Fisch et al., 1998).

A Figura 4 apresenta o espectro de potência médio dos coeficientes da TWM em porcentagem e em função dos períodos em dias, para os 11 anos, que mostra a energia integrada em todo o tempo para um determinado período. As porcentagens de potência representada pela oscilação anual, seguida pela interanual e semi-anual foram submetidas ao teste de significância apresentado por Torrence e Compo (1998) e são significativas a $95 \%$.

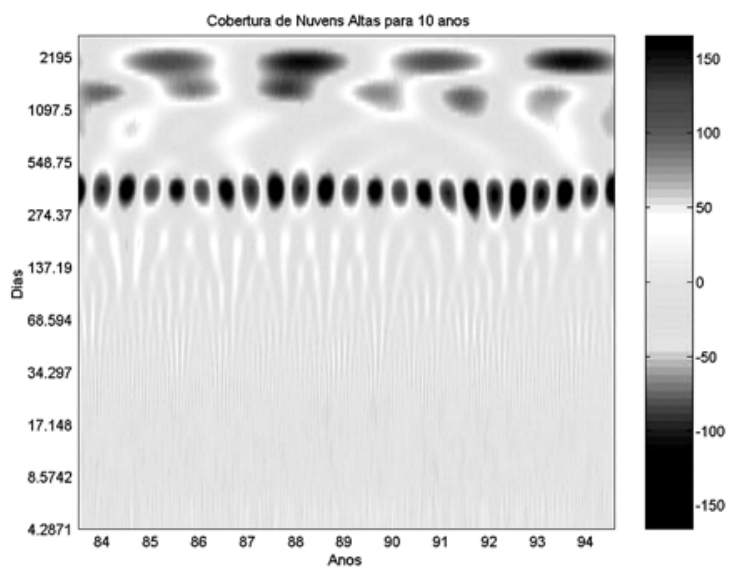

Figura 3 - Parte real da Transformada Wavelet de Morlet aplicada à série temporal da cobertura de nuvens altas às 00:00 UTC, para um setor de 2,50 $x 2,5^{\circ}$, centrado em Manaus. Na ordenada são apresentados os anos analisados e na abscissa o período em dias.

\section{Espectro de potência da wavelet}

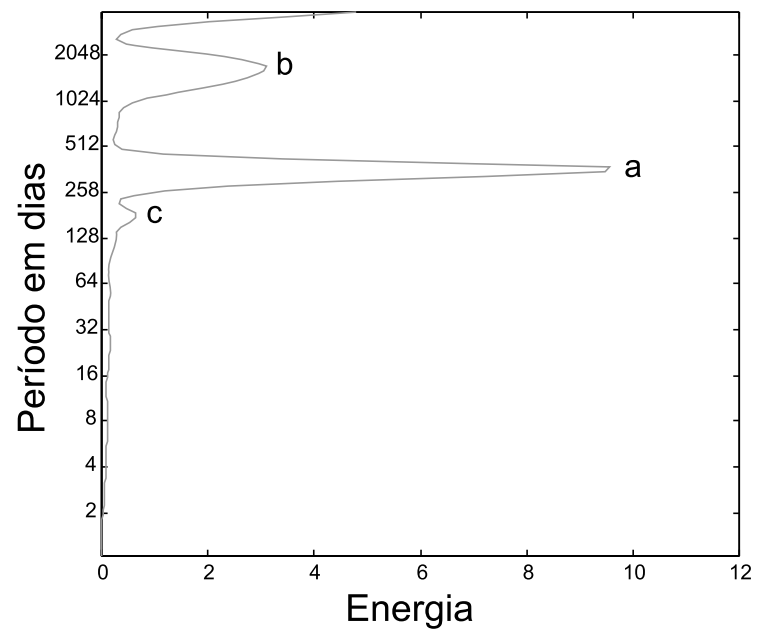

Figura 4 - Espectro da porcentagem de potência médio dos coeficientes da TWM em função dos períodos em dias para os 11 anos. Sendo que: (a) oscilação anual -365 dias, (b) oscilação interanual -1536 dias e (c) oscilação semi-anual-193 dias.
Para identificar as principais oscilações da cobertura de nuvens e verificar se existem variações dessas oscilações em anos de El Niño (que seriam os anos secos) e La Niña (anos chuvosos), inicialmente, faz-se uma comparação das médias de cobertura de nuvens em anos secos e chuvosos. Pelo teste t-Student a $1 \%$, as médias são diferentes (significância de 99\%). Então, calcula-se o espectro de potência médio dos anos chuvosos (anos que possuem desvio acima da média) e dos anos secos (desvios abaixo da média). É utilizado como referência o ano hidrológico, isto é, os anos começando em julho e terminando em junho do ano seguinte. Para o espectro de potência médio dos anos chuvosos, utilizamse os seguintes anos: $85 / 86,86 / 87$ e 89/90; para os anos secos: 83/84, 87/88 e 92/93. Os anos de 84/85, 88/89, 90/91 e 91/ 92 não são utilizados, pois são anos de transição entre os desvios positivos e negativos.

Para se estudar a variabilidade destes espectros ano a ano, associa-se a cada período a média, o maior e o menor valor de potência. A Figura 5 apresenta o espectro da porcentagem de potência médio dos coeficientes da TWM em função dos períodos em dias para os anos secos e chuvosos. Nesta figura, observa-se que a oscilação anual dos anos secos possui maior porcentagem de potência que os chuvosos. O inverso é observado para a oscilação semi-anual, que possui maior porcentagem de potência para os anos chuvosos que para os secos. Devido à pequena diferença existente entre as porcentagens de potência, verificou-se que as diferenças não são estatisticamente significativas. Essa diferença deve estar associada ao reduzido número de anos utilizados neste estudo.

$\mathrm{Na}$ Figura 6, apresenta-se o espectro de porcentagem de potência médio dos coeficientes da TWM, integrando somente

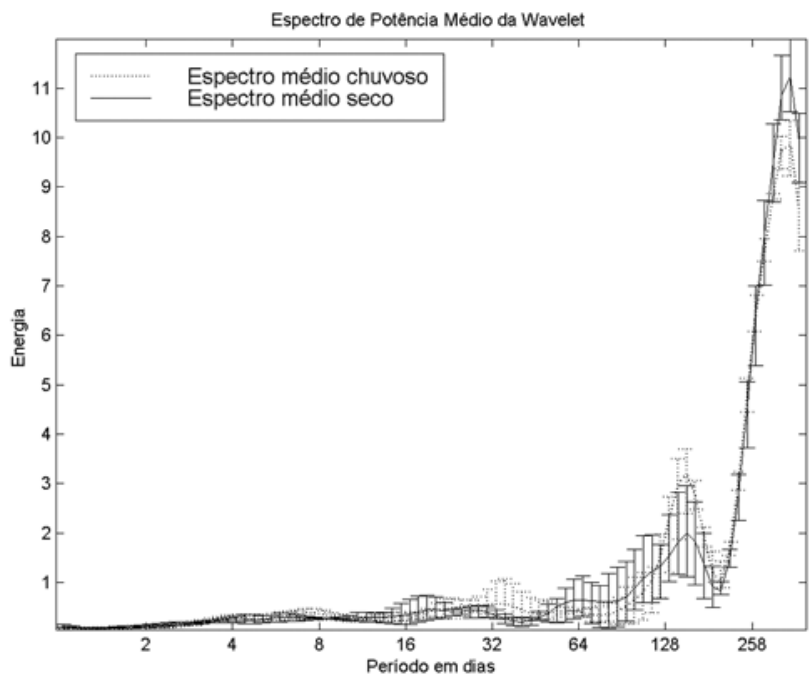

Figura 5 - Espectro da porcentagem de potência médio dos coeficientes da TWM em função das freqüências em dias para os anos chuvosos e secos. As barras correspondem ao maior e menor valor encontrado nas séries individuais de cada ano. 


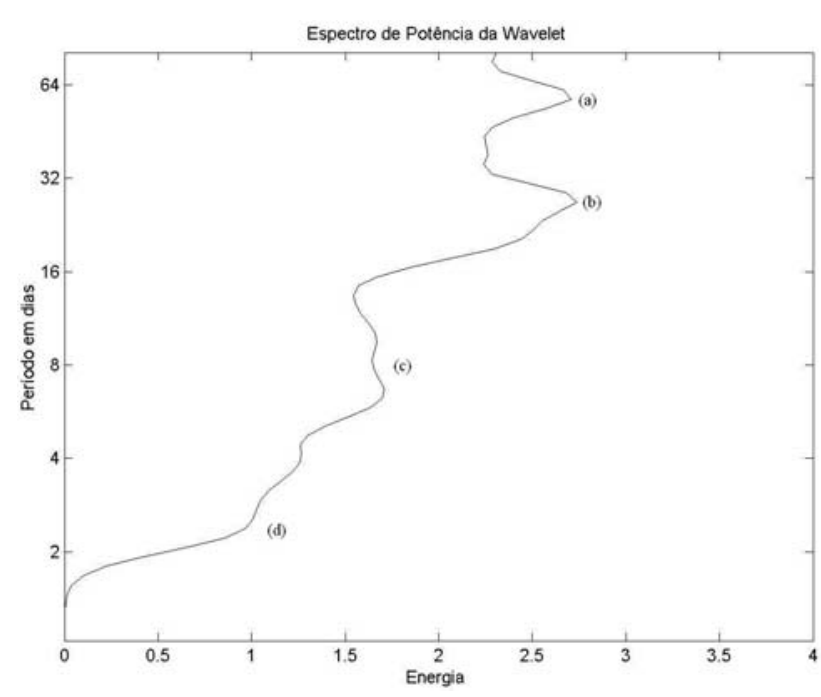

Figura 6 - Espectro da porcentagem de potência média dos coeficientes da TWM dos períodos em dias para os 11 anos. Sendo que: (a) oscilação - 60 dias, (b) oscilação-30 dias, (c) oscilação interdiurna - (11-7) dias, (d) oscilação interdiurna -3 dias.

os períodos menores, isto é, de 1 até 70 dias. Desse modo, observase, mais claramente, a potência das oscilaçôes intra-sazonal (6030) dias e interdiurnas (11-3) dias. Os resultados das oscilaçōes interdiurnas também encontrados por Machado et al. (1999), os quais verificaram que as propagações meridionais de convecção na Amazônia apresentam periodicidade em torno de 3 a 6 dias e estão associadas às penetraçôes de sistema frontais. Estes sistemas frontais possuem uma forte variabilidade em torno de 7 dias (Oliveira, 1986).

Quanto à oscilação intra-sazonal, Knutson e Weickmann (1987) verificaram que, nos trópicos, as anomalias do vento zonal na baixa e alta troposfera estão fora de fase na escala de tempo de 30-60 dias. Kayano et al. (1990) observaram que as chuvas, principalmente no nordeste, são influenciadas pela oscilação 3060 dias, enquanto Nogués-Paegle e Mo (1997), estudando variações na formação da ZCAS, encontraram resultados indicando que a convecção da ZCAS poderia estar sendo influenciada pela oscilação de 30-60 dias.

Para verificar se existem diferenças nos espectros de potência para as oscilaçōes intra-sazonal e interdiurnas em anos de El Niño e La Niña, calcula-se o espectro da potência média integrado no intervalo de 1 a 78 dias (Figura 7). Observa-se que os anos chuvosos apresentam máximos secundários para os períodos de 32, 8 e 4 dias, e os anos secos apresentam máximos secundários nos períodos de 64, 20 e 6 dias. As diferenças de potência entre os anos secos e chuvosos não são estatisticamente significativas a um nível de teste de 5\%. A não significância destas diferenças deve provavelmente estar associada à reduzida população de anos utilizados neste estudo.

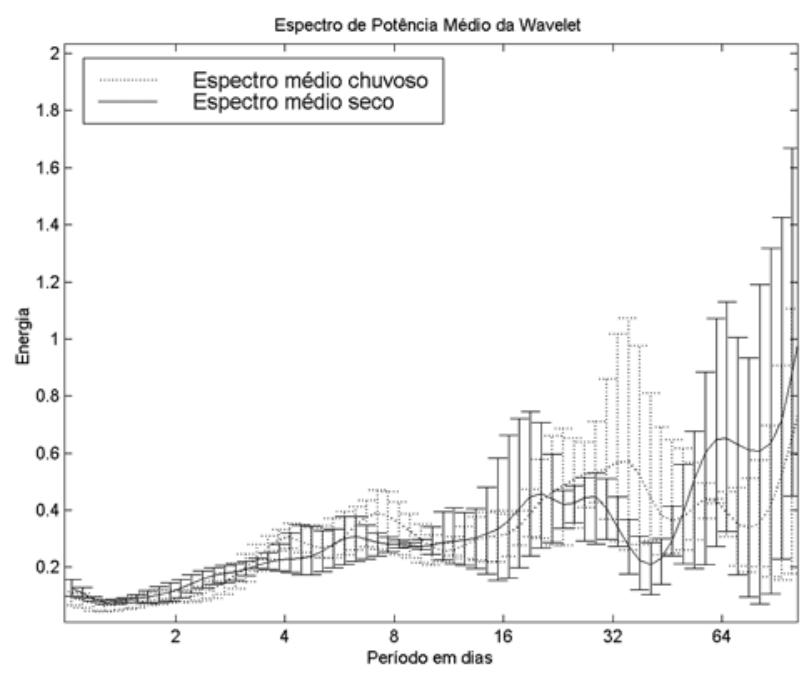

Figura 7 - Espectro da porcentagem de potência médio dos coeficientes da TWM em função dos períodos em dias (1 a 78 dias) para os anos chuvosos e secos. As barras correspondem ao maior e menor valor encontrado nas séries individuais de cada ano.

Para estudar os locais onde as oscilações de um dado período são mais intensas, analisa-se a região compreendida pelas coordenadas $1,5^{\circ} \mathrm{S}$ a $6^{\circ} \mathrm{S}$ e $68^{\circ} \mathrm{W}$ a $54^{\circ} \mathrm{W}$, que abrangem 21 setores, centrados em Manaus. Considerando-se as oscilações com períodos mais intensos, calcula-se a porcentagem de potência para todos os setores (Figura 8) e observa-se que os períodos de 60, 45 e 30 dias possuem maior porcentagem de potência a leste de Manaus; e para o período de 60 dias também mostra um máximo no centro da grade. $O$ período de 20 dias possui maior energia no centro (próximo de Manaus); e os períodos de 8, 6 e 4 dias possuem maior energia a oeste de Manaus. Este resultado está de acordo com os obtidos por Kayano et al. (1990), Nascimento \& Brito (2002), os quais afirmaram que a oscilação de 30-60 dias atua principalmente no Nordeste brasileiro. Oscilaçôes interdiurnas, ao redor de cinco dias, foram observadas por Machado et al. (1996), Guedes e Machado (1998) e Siqueira \& Machado (2004), estas podem estar associadas com a penetração de sistemas frontais.

Utilizando a metodologia de Siqueira e Machado (2004) para detecção de frentes frias, define-se uma janela de $10^{\circ}$ de longitude $\left(48,75^{\circ}\right.$ a $\left.58,75^{\circ} \mathrm{W}\right)$ e $2,5^{\circ}$ de latitude $\left(5,0\right.$ a $\left.7,5^{\circ} \mathrm{S}\right)$. Determinou-se o dia médio da entrada das frentes frias que alcançaram esta janela. Para um dado dia médio, encontra-se o período correspondente à maior amplitude de potência (obtida pela análise de Wavelet). Usando este procedimento, são estudados 440 casos, divididos conforme a estação do ano. A Tabela 1 mostra que as frentes frias que alcançam a janela definida acima modulam de alguma forma, a atividade convectiva na região de Manaus atuando como uma forçante das oscilações interdiurnas 
(a)

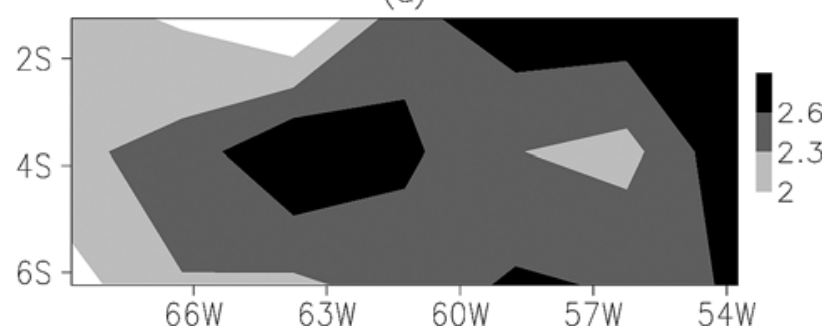

(b)

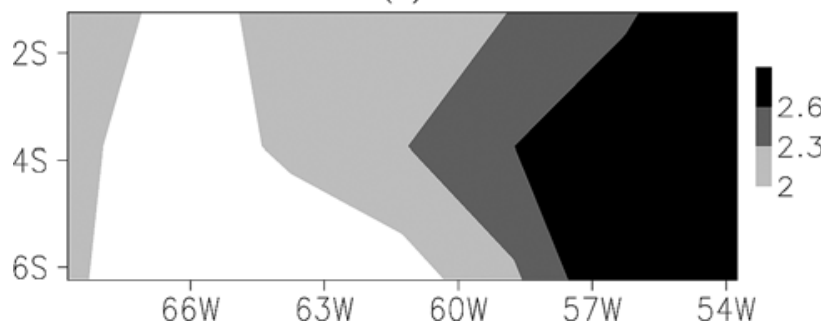

(c)

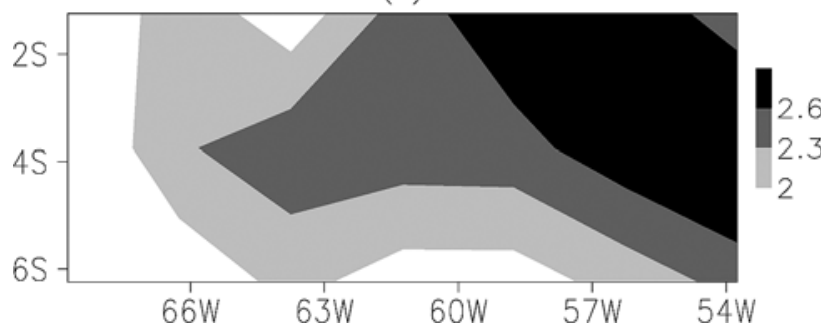

(d)

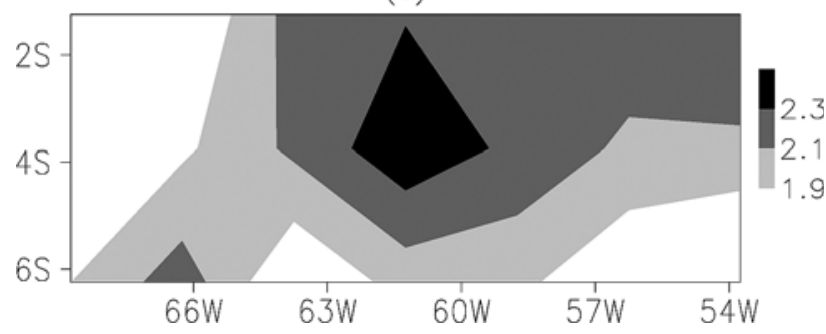

Tabela 1 - Porcentagem da entrada de frentes frias (dia médio) com a correspondente amplitude máxima obtida pela Wavelet para 0 dia médio, associado ao período.

\begin{tabular}{ccccc}
\hline Frentes & $\mathbf{2 - 4}$ dias & $\mathbf{5 - 1 4}$ dias & $\mathbf{2 5 - 3 5}$ dias & 55-65 dias \\
\hline DJF & 5,2 & 22,3 & 24,1 & 15,6 \\
MAM & 12,6 & 48,6 & 10,9 & 7,2 \\
JJA & 24,5 & 28,9 & 4,5 & 8,9 \\
SON & 14,6 & 31,4 & 21,2 & 18,9 \\
MÉDIA & 14,3 & 32,8 & 15,3 & 12,6 \\
\hline
\end{tabular}

(e)

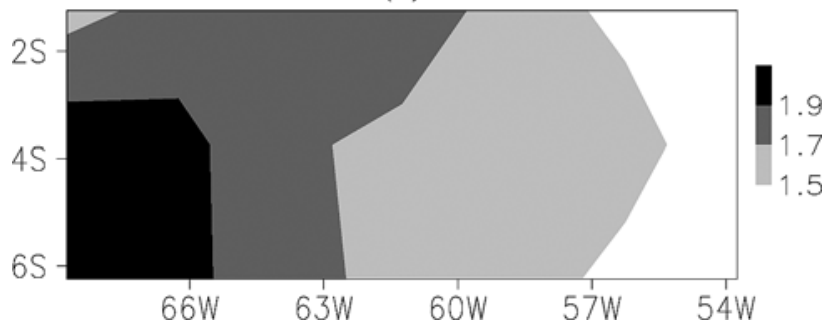

$(f)$

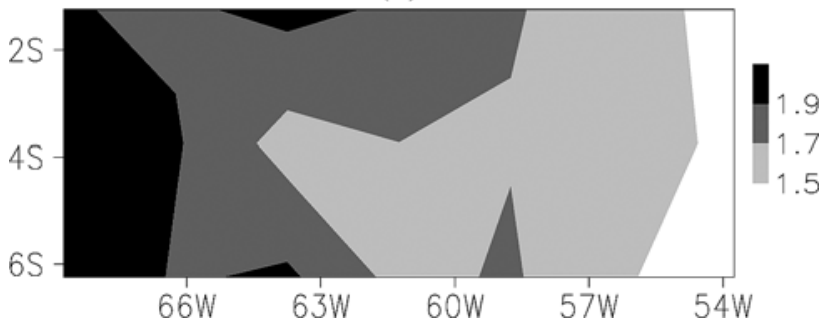

(g)

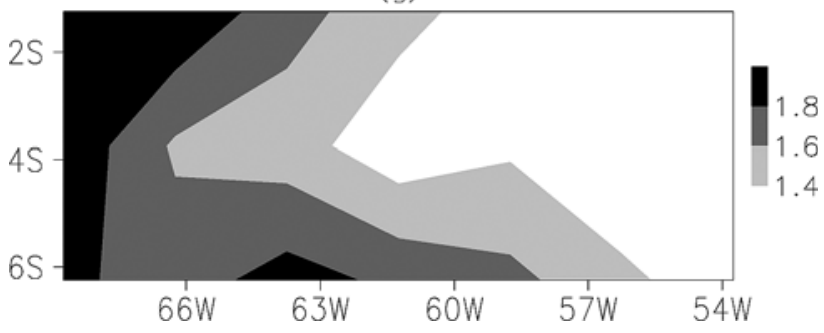

Figura 8 - Porcentagem de potência para uma região compreendida de $1,5^{\circ} \mathrm{S}$ a $6^{\circ} \mathrm{S}$ e $68^{\circ} \mathrm{W}$ a $54^{\circ} \mathrm{W}$, para as oscilações: (a) 60 , (b) 45 , (c) 30 , (d) 20, (e) 8, (f) 6, e (g) 4 dias.

em torno de $47 \%(14,3 \%+32,8 \%)$ dos casos, das oscilações intra-sazonais com períodos entre 25 - 35 dias (15\%), e das oscilaçōes com períodos entre 55 a 65 dias (13\%). Observa-se ainda que este efeito seja também modulado sazonalmente. Notase que flutuaçōes em torno de 2 a 4 dias predominam nos meses de junho a agosto, e de 5 a 14 dias nas pré-estaçōes seca e chuvosa. Os períodos entre 25 a 65 dias atuam mais fortemente na préestação chuvosa e na estação chuvosa. Convém ressaltar que não necessariamente as frentes frias que causaram as oscilações. As frentes podem favorecer ou induzir a convecção em grande parte da Amazônia. Figueroa \& Nobre (1990) observaram que parte da precipitação na região Central da Amazônia (próximo de 5S) pode estar associada com penetração de sistemas frontais da região sul, interagindo e organizando a convecção. 
A ação de sistemas frontais induzindo oscilaçōes interdiurnas está em parte relacionada com o fenômeno de friagem. Marengo et al. (1997) observaram a ação eventual de sistemas frontais na Amazônia, provocando o fenômeno localmente denominado de friagem, que durante os anos de 1992/1993 obteve uma freqüência de ocorrência de 7 casos no ano, com duração de 3 a 5 dias nos meses de maio a agosto na Amazônia Central.

Quanto à oscilação intra-sazonal, Castro (2002) verificou que há indícios da existência de uma periodicidade de 20 dias nos eventos da ZCAS e segundo seus resultados, as ZCAS que se formam mais próximo ao equador recebem uma influência direta da oscilação de 30-60 dias. Nogués-Paegle e Mo (1997) utilizando radiação de onda longa e produtos de reanálises do NCEP descrevem períodos de abundância e de deficiência de precipitação na América do Sul durante o verão, de acordo com a variação da ZCAS que poderia ter uma ligação com a oscilação de 30-60 dias.

Por um lado, as oscilações intra-sazonais normalmente associadas à oscilação de escala planetária conhecida como oscilação de Madden-Julian, têm sido relacionadas às mudanças na posição e intensidade da ZCAS (Casarin \& Kousky, 1986). Por outro lado, a ZCAS está associada à penetração de sistemas frontais e a conseqüente associação com a convecção tropical. Seria a oscilação 30-60 dias, na Amazônia, conseqüência de uma interação entre as oscilações em escala planetária, penetração de sistemas frontais e ZCAS? Essa é uma questão que deve ser verificada com um conjunto maior de dados.

Embora a energia associada à oscilação de 30 e 60 dias possa ser considerada baixa em relação a toda variabilidade do espectro, ela é importante porque deve interagir com outras escalas. $\mathrm{Ou}$ seja, o conhecimento das características das oscilações das nuvens altas pode adicionar mais informação no entendimento da física envolvida na modulação da cobertura de nuvens na região Amazônica.

\section{CONCLUSÕES}

Neste trabalho utilizam-se análises espectral e transformada de wavelet no período de 12 anos (1983-1994). Estes dados foram extraídos das imagens de satélites do ISCCP, com o objetivo de detectar as oscilaçóes que atuam na cobertura de nuvens sobre a Amazônia Central. Os maiores desvios interanuais foram obtidos para a cobertura de nuvens altas, isso indica que os processos associados à convecção são aqueles que mais sofrem influências interanuais.

O período predominante corresponde à oscilação anual. As oscilaçôes interanuais apresentam forte variabilidade da cobertura de nuvens, e devem estar associadas a episódios de El Niño e La Niña. As oscilaçóes intra-sazonais e interdiurnas apresentam uma variabilidade menor. As diferenças entre os espectros de potência médio chuvoso e seco das oscilações interdiurnas e intra-sazonais não são significativas, isto devido à reduzida população de anos utilizados neste estudo.

Considerando a influência de sistemas frontais na Amazônia, verifica-se que as variações mais significativas na cobertura de nuvens centrada na alta freqüência (4, 6 e 8 dias) ocorrem na porção ocidental da Amazônia (a oeste de Manaus); enquanto que as variações na baixa freqüência (escalas intra-sazonais de 30 e 45 dias) se processam na porção oriental da Amazônia (a leste de Manaus). Logo, pode-se concluir que as frentes frias que alcançam a janela de $10^{\circ}$ de longitude e $2,5^{\circ}$ de latitude (centrada em Manaus), modulam de alguma forma a atividade convectiva na região de Manaus, atuando como uma forçante das oscilações interdiurnas em torno de $47 \%$ dos casos, das oscilações intrasazonais com períodos entre 25 - 35 dias (15\%), e das oscilaçóes com períodos entre 55 a 65 dias (13\%). Observa-se que este efeito é também modulado sazonalmente. Nota-se que flutuações em torno de 2 a 4 dias são predominantes nos meses de junho a agosto, e de 5 a 14 dias nas pré-estações seca e chuvosa. Os períodos entre 25 a 65 dias atuam mais fortemente na pré-estação chuvosa e na estação chuvosa.

Convém ressaltar que não necessariamente as frentes frias causaram as oscilaçôes. As frentes podem favorecer ou induzir a convecção em grande parte da Amazônia diretamente ou indiretamente. $\mathrm{O}$ conhecimento das características das oscilações das nuvens altas em diversas escalas temporais contribui para um melhor entendimento da modulação da cobertura de nuvens na regiāo Amazônica.

\section{AGRADECIMENTOS}

Os autores agradecem o apoio dos pesquisadores José Ricardo Siqueira, Marcos D. Oyama e Roberto Lage Guedes. Agradecem também as sugestōes e correçōes dos revisores anônimos.

\section{BIBLIOGRAFIA CITADA}

Andreoli, R.V.; Kayano, M.T.; Guedes, R.L.; Oyama, M.D.; Alves, M.A.S. (2004). A Influência da Temperatura da Superfície do Mar dos Oceanos Pacífico e Atlântico na Variabilidade de Precipitação em Fortaleza. Rev. Bras. Meteo, 19(3): 337-344.

Castro, C.A.C. 2002. Interaçôes Trópicos-Extratrópicos na escala de tempo intra-sazonal durante o verão austral e seus efeitos na América do Sul. Dissertação de Mestrado. Instituto Nacional de Pesquisas Espaciais, São Paulo.

Casarin, D.P.; Kousky, V.E. 1986: Precipitation anomalies in the southern part of Brazil and variations of the atmospheric circulation. Rev. Bras. Meteor, 1:83-90.

Chapa, R.S.; Rao, V.B.; Prasad, G.S.S.D. 1998. Application of Wavelet Transform to Meteosat-derived cold cloud index data over South America. Mon. Wea. Rev, 126: 2466-2481.

Cavalcanti, I.F.A. 1996. Episódios El Niño/Oscilação Sul durante a década de 1986 a 1996 e Suas Influências sobre o Brasil. Climanálise 10 anos, CPTEC/INPE. 
Coelho, C.A.S.; Drumond, A.R.M.; Ambrizzi, T. 1999. Estudo Climatológico Sazonal da Precipitação Sobre o Brasil em Episódios Extremos da Oscilação Sul. Rev. Brás. Meteo. 14(1): 49-65.

Cutrim, E.M.C, Molion, L.B.; Nechet, D. 2000. Chuvas na Amazônia Durante o Século XX. Anais: XI Congresso Brasileiro de Meteorologia. Rio de Janeiro. 1327-1333.

Farge, M. 1992: Wavelet transforms and their applications to turbulence. Annu. Rev.Fluid Mech., 24:395-457.

Figueroa, S.N; Nobre, C.A. 1990. Precipitations Distribution Over Central and Western Tropical South America. Climanálise, 5 (6): 36-45.

Fisch, G.; Marengo, J.A.; Nobre, C.A. 1998. Uma Revisão Geral sobre o Clima da Amazônia. Acta Amazonica, 28(2): 101-126.

Guedes, R.L.; Machado, L.A.T. 1997. Características Médias da Cobertura de Nuvens sobre a América do Sul com Base em Imagens do GOES-E/ISCCP: julho de 1987 a junho de 1988. Rev. Bras. Meteo, (12): 1-19.

Guedes, R.L.; Machado, L.A.T. 1998. Características da Propagação dos sistemas convectivos no oeste da Amazônia. Anais X Congresso Brasileiro de Meteorologia. Brasilia. CD- MS 98013.

Kayano, M.T.; Kousky, V.E.; Studzinski, C.D.; Dias, P.L.S. 1990. As Variações Intra-Sazonais da Precipitação no Brasil durante o Verão de 1989/1990. Climanálise, 5 (4): 40-50.

Kayano, M.T.; Kousky, V.E. 1992. Sobre Monitoramento das oscilações intra-sazonais. Rev. Bras. Meteo, 7(2):593-602.

Kayano, M.T.; Kousky, V.E. 1999. Intraseasonal (30-60 day) variability in global tropics: principal modes and their evolution. Tellus, 51A, 373-386.

Kousky, V.E., 1987. The Global Climate for December 1986February 1987: El Niño returns to the tropical Pacific. Mon. Wea. Rev, 115, 2822-2838.

Machado, L.A.T.; Guedes, R.L.; Alves, M.A.S. 1996. Características Estruturais de Sistemas Convectivos e Forçantes na América do Sul Observados por Satélites. Climanálise 10 anos, CPTEC/ INPE, 110-122, S.P.

Machado, L. A. T.; Duvel, J. P.; Laurent, H.; Siqueira, J. R.. 1999. Meridional propagation of the convection in South America. $6^{\circ}$ International Conference on Southern Hemisphere Meteorology and Oceanography, Montevideu, Chile, Amer. Meteor. Soc., 398399.

Madden, R.A.; Julian, P. R. 1994: Observations of the 40-50 day tropical oscillation -A Review. Mon. Wea. Rev. (122): 814-837.

Marengo, J.; Nobre, C.A.; Culf, A.D. 1997. Climatic impacts of the "Friagens" in forested and deforested areas of the Amazon Basin. Journal of Climate, 36(11): 1553-1566.

Marengo, J.A.; Liebmann, B; Kousky, V.E.; Filizola, N.P.; Wainer, I.C. 2001: Onset and end of the rainy season in the Brazilian Amazon Basin. J. Climate, (14): 833-852.

Mattews, A.J. 2000. Propagation mechanisms for the Madden-Julian Oscilation. Q.J.R.M.S., 126(569): 2637-2652.
Nascimento, W.A.; Brito, J.I.B. 2002 . Verificação da Atuação de Oscilação de 30-60 dias no Nordeste do Brasil. XII Congresso Brasileiro de Meteorologia. Foz do Iguaçu-PR.

Noguès-Paegle, J.; Mo, K.C. 1997. Alternating wet and dry conditions over South America during summer. Mon. Wea. Rev., (125): 279-291.

Molion, L.C.B. 1987. Climatologia Dinâmica da Regiāo Amazônica: mecanismos de precipitação. Rev. Bras. Meteo, 2(1): 107-117.

Oliveira, A.S. 1986. Interaçôes entre Sistemas Frontais na América do Sul e Conveç̧ão na Amazônia. INPE-4008-TDL/239.

Oliveira, G.S. 1999. O El Niño e Você: O Fenômeno Climático, Transtec Editorial. 115pp.

Rao, V.B.; Hada, K. 1990. Characteristic of Rainfall over Brazil: Anual Variation and Connections with the Southern Oscillation. Theor. Appl. Climatol, (42): 81-91.

Oliveira, L.L.; Vianello, R.L.; Ferreira, N.J. 2001. Meteorologia Fundamental, Edifapes, 430pp.

Rossow, W.B.;Walker, A.W. 1991. International Satellite Cloud Climatology Project (ISCCP). Description of Monthly Mean Cloud Data (Stage C2). Apendix C, WMO/ICSU.

Rossow, W.B.; Garder, L.C.; Lu, P.J.; Walker, A. 1991. International Satellite Cloud Climatology Project (ISCCP). Documentation of Cloud Data. WMO/ICSU, No 266.

Schiffer, R. A.; Rossow, W. B. 1983. The International Satellite Cloud Climatology Project (ISCCP): The first Project of the World Climate Research Programme. Bull. Amer. Met. Soc., 64: 779-784.

Siqueira, J.R.; Machado, L.A.T. 2000. Variações Interanuais das Trajetórias das Perturbações Sinóticas na América do Sul e Áreas Adjacentes. Anais: XI Congresso Brasileiro de Meteorologia. Rio de Janeiro.

Siqueira, J.R.; Machado, L.A.T. 2004. Influence of the Frontal Sytems on the day-to-day Convection Variability over South América. J. Climate, 17: 1754-1766.

Torrence, C.; Compo, G. P. 1998. A Practical Guide to Wavelet Analysis. Bulletin of the American Meteorological Society, 79: 6178.

Weng, H.; Lau, M.K. 1994. Wavelets, Periods Doubling and Time Frequency Localization with Application to Organization of Convection over the Tropical Western Pacific. J. Atmos. Sci., 51: 2523-2541.

Knutson, T.R.; Weickmann, K.M. 1987: 30-60 day Atmospheric Oscillations: Composite Life Cycles of Convection and Circulation Anomalies. Mon. Wea. Rev., 115: 1407-1436.

Vianello, R. L.; Alves, A. R.1991. Meteorologia Básica e Aplicações. 449pp.

Recebido em 02/06/2003

Aceito em 23/01/2007 
